\title{
Hydrogel Microspheres II. Precipitation Copolymerization of Acrylamide with Comonomers to Prepare Monodisperse Hydrogel Microspheres
}

\author{
Haruma Kawaguchi, Yukino Yamada, Shinobu Kataoka, \\ Yasuhiro MoRITA and Yasuji OHTSUKA \\ Department of Applied Chemistry, Faculty of Science \& Technology, Keio University, \\ 3-14-1 Hiyoshi, Kohoku-ku, Yokohama 223, Japan
}

(Received December 11, 1990)

\begin{abstract}
Precipitation polymerization of acrylamide (AAm) and methylene-bisacrylamide (MB) was carried out in alcohols. Large, bulky and porous particles were formed by the polymerization. On the contrary, copolymerization of AAm and MB with a certain amount of methacrylic acid (MAc) resulted in the formation of fine monodisperse hydrogel microspheres. This was attributed to the contribution of MAc units to the stabilization of the particles formed at the initial stage of polymerization and the enhancement of swelling of the particles by monomer and alcohol.
\end{abstract}

KEY WORDS Hydrogel / Microsphere / Precipitation Polymerization Polyacrylamide / Monodisperse Particle

Polyacrylamide (Poly-AAm) microspheres have been prepared by inverse emulsion or suspension polymerizations. ${ }^{1-4}$ Any of these polymerizations did not give monodisperse microspheres and the products were contaminated with emulsifiers and costabilizers. In this study, precipitation polymerization of AAm was examined to prepare monodisperse and contaminant-free hydrogel microspheres.

Precipitation polymerization is defined as a polymerization starting from homogeneous monomer solution in which the polymer is insoluble. Polymers precipitate in the course of polymerization and usually form coagulum or aggregates instead of fine microspheres. But, recently a few exceptional cases were found out by Pelton ${ }^{5}$ and us $^{6}$ in the polymerizations of some $N$-substituted AAms, in which monodisperse hydrogel microspheres were obtained. In precipitation polymerization of AAm and some comonomers, it was found that methacrylic acid (MAc) as a comonomer was very effective in making the microspheres fine and monodisperse. This is a new finding so far as I know. Therefore, the role of MAc was studied and the mechanism of particle formation was discussed.

\section{EXPERIMENTAL}

\section{Materials}

Acrylamide (AAm) was recrystallized from acetone. Methylenebisacrylamide (MB) was used as received. Methacrylic acid (MAc) was distilled under a reduced pressure. Azobisisobutyronitrile (AIBN) and azobis(4-cyanopentanoic acid) (V-501, Wako Pure Chemicals Co.) were used as initiators. AIBN was precipitated from chloroform solution with methanol. V-501 was used without further purification. Three kinds of alcohols, methanol, ethanol, and $n$-propanol were distilled before use.

\section{Polymerization}

Polymerizations were carried out in a $100 \mathrm{ml}$ 
round-bottom flask at $60^{\circ} \mathrm{C}$ under nitrogen. In $0.025 \mathrm{~g}$ initiator were used. The ratio of standard recipes, $50 \mathrm{~g}$ solution of monomer and monomer to solvent was changed from $1 / 49$ to

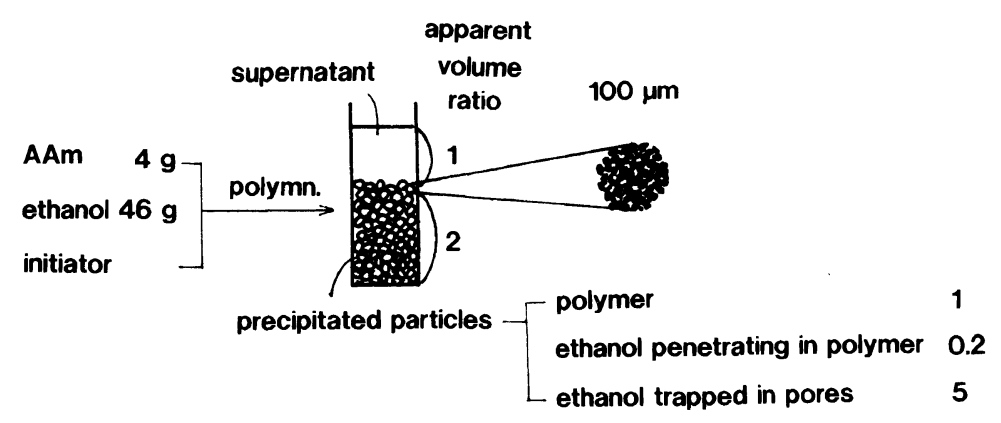

Figure 1. Typical polymerization recipe and construction of the products.

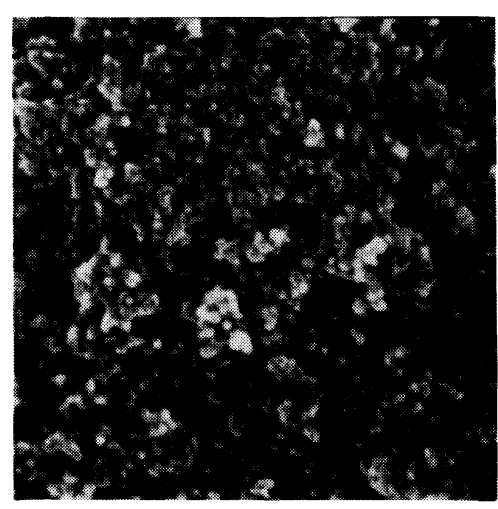

conv. $5 \%$

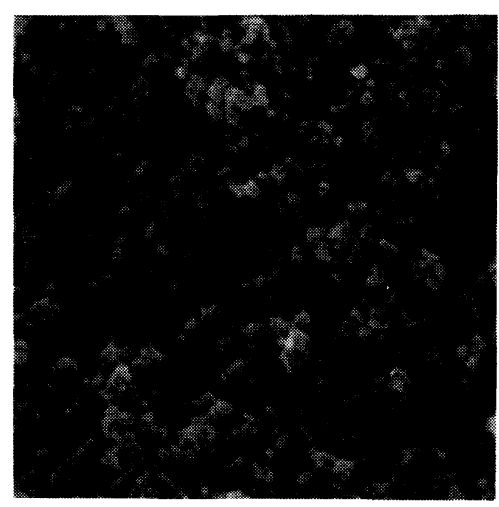

conv. $38 \%$

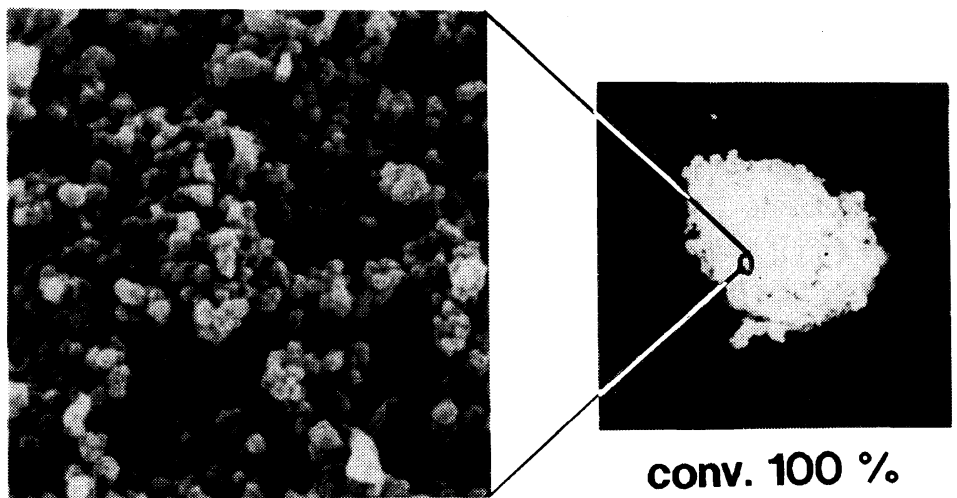

conv. $100 \%$

\section{$1 \mu \mathrm{m}$}

Figure 2. SEM views of the precipitates at different conversions in the precipitation polymerization of AAm in ethanol. Polymerization: AAm/ethanol/AIBN=2/48/0/025 (g). 
$8 / 42(\mathrm{~g} / \mathrm{g})$. The monomer composition, AAm/ $\mathrm{MB} / \mathrm{MAc}$, was from $1 / 0 / 0$ to $1 / 1 / 2$. The polymerizations were carried out for 8 hours unless otherwise noted.

\section{Measurements}

The conversion was measured by gravimetry. The size and its distribution and the shape of polymer particles were observed on the electron micrographs. The average hydrodynamic size was determined by photon correlation spectroscopy when the microspheres had narrow size-distribution. The molecular weight was measured by viscometry or gel permeation chromatography as a function of conversion.

The extent of swelling of crosslinked poly-AAm and poly(AAm-co-MAc) by alcohol was determined using crosslinked polymer films. The films were immersed in ethanol and kept at $60^{\circ} \mathrm{C}$ overnight. The increment in weight was measured to calculate the extent of swelling. The polymer films were also immersed in a ethanol solution of AAm monomer to determine the partition of AAm between the polymer phase and continuous phase.

\section{RESULTS AND DISCUSSION}

\section{Precipitation Polymerization of Acrylamide}

Precipitation polymerization of AAm was carried out in three kinds of alcohols, methanol, ethanol and $n$-propanol using two kinds of initiators, AIBN and V-501. The polymerization rate was influenced by the sort of alcohols as well as initiators. The dependence of molecular weight on conversion differed among the systems of different media and initiators although no Tromsdorff effect was observed in any systems. The details of polymerization kinetics will be discussed in our another paper.

The products of polymerizations were commonly coarse and bulky particles having a diameter about $100 \mu \mathrm{m}$. They were aggregates of primary particles independent of the sorts of alcohols and initiators used. The appearance of the products resembled that in the suspension polymerization of vinyl chloride, in which polymer precipitates in monomer droplets. ${ }^{7,8}$ DeSchryver et al. did a preliminary investigation on the precipitation polymerization of AAm in alcohol. They must have had polymer aggregates, but they paid no attention on the size and morphology of the products. ${ }^{9}$

The apparent volume of particles was very large as shown in Figure 1. Namely, the apparent volume fraction of precipitated particle phase in the product was about two thirds whereas the polymerization system included only $4 \mathrm{~g}$ monomer in $50 \mathrm{~g}$ solution. This means that the particles contain a large amount of solvent inside them. It was found that only a small fraction of such solvent dissolved in poly-AAm to swell it and the rest, a large fraction of the solvent, was just trapped in the pores of particles. The fraction of each solvent is shown in Figure 1.

According to the observation of growing particles with an electron microscope, shown in Figure 2, small microspheres (primary particles) formed continuously even in the later stage of polymerization and they aggregated into coarse particles. The picture at the right bottom of Figure 2 shows a coarse particle.

It is speculated from these results that the particles are formed in the manner shown in

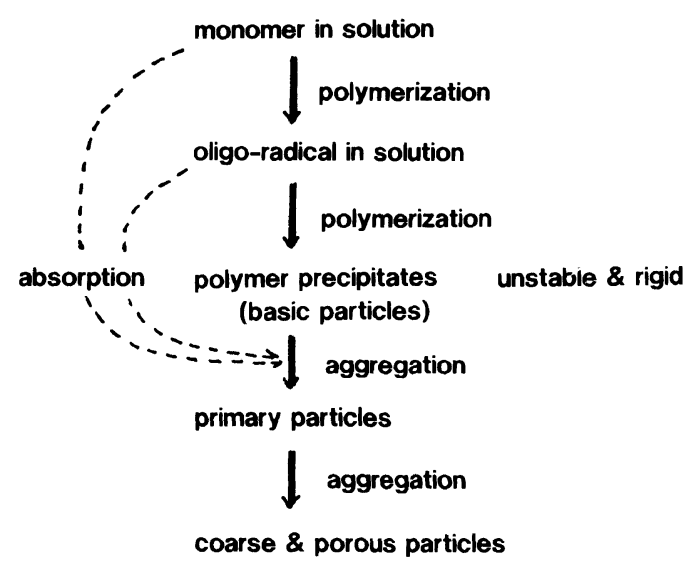

Figure 3. Scheme for particle growth in the precipitation polymerization of AAm in ethanol. 


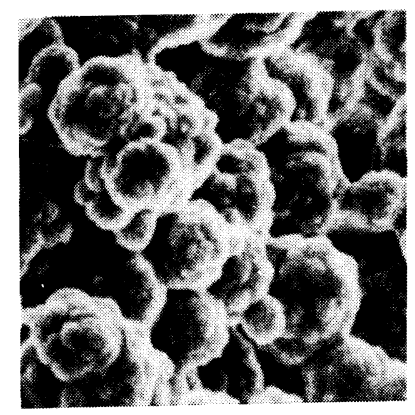

a

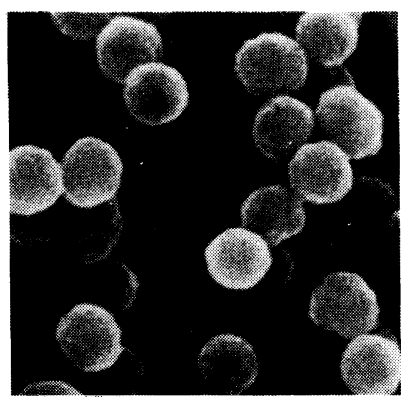

C

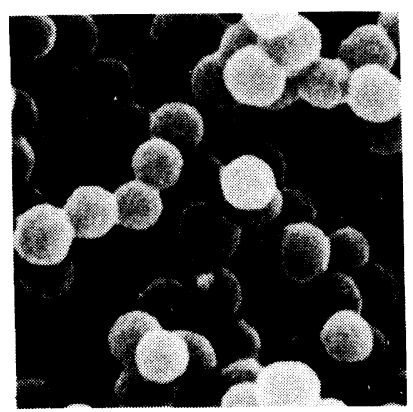

e

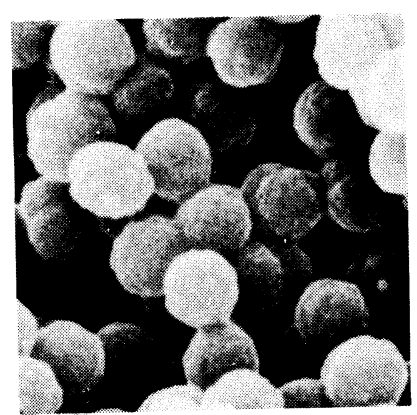

b

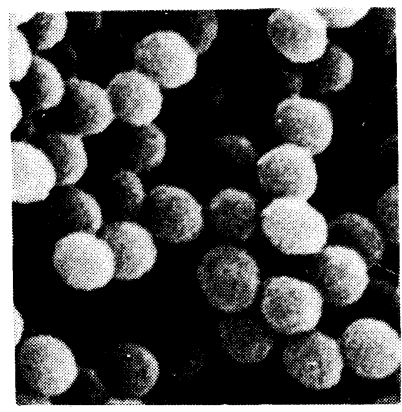

d

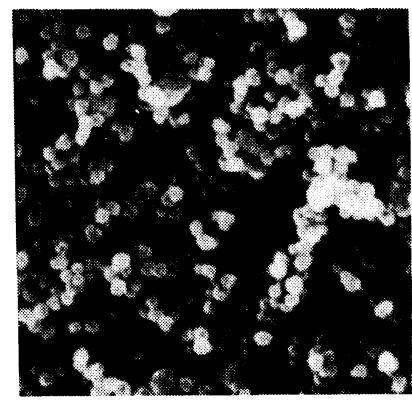

f

$1 \mu \mathrm{m}$

Figure 4. SEM views of polymer particles formed by precipitation copolymerization of AAm, MB, and MAc in ethanol.

\begin{tabular}{ccccccc}
\hline & $\mathrm{a}$ & $\mathrm{b}$ & $\mathrm{c}$ & $\mathrm{d}$ & $\mathrm{e}$ & $\mathrm{f}$ \\
\hline $\mathrm{AAm} / \mathrm{MB} / \mathrm{MAc}(\mathrm{g})$ & $6 / 1 / 1$ & $5 / 1 / 1$ & $4 / 1 / 1$ & $3 / 1 / 1$ & $2 / 1 / 1$ & $1 / 1 / 1$ \\
\hline
\end{tabular}


Figure 3. The figure was drawn by refering to the scheme for the formation of poly(vinly chloride) granules. ${ }^{10}$ According to Diamadopoulos, et al., ${ }^{10}$ the poly(vinyl chloride) granules are formed by the combination of the following processes: a) the formation of polymer molecules due to solution polymerization; b) the separation of the polymer phase from the continuous phase with the formation of insoluble polymer particles (basic particles having a diameter around $20 \mathrm{~nm}$ ); c) the aggregation of the basic particles to form primary particles (diameter of $100-200 \mathrm{~nm}$ ), and d) the aggregation of primary particles accompanying the growth of the particle volume due to the polymerization in the polymer phase. The size and individuality of the primary particles depend on their stability and the partition of monomer between continuous and polymer phases. In the polymerization of vinyl chloride, an appreciable electrostatic repulsive force is believed to work between particles to disperse the particles stably. ${ }^{8,10}$ In the precipitation polymerization of AAm in alcohols, the polymer precipitates or basic particles are unstable because of lack of electrostatic and steric repulsive forces. In addition to this, the monomer dissolves little in the polymer. Therefore, it is probable that polymerization in the precipitated polymer phase plays a little role in the growth of primary particles and, therefore, the polymerization finally gives coarse and porous particles, which are aggregates of primary particles, mainly via pathway shown with solid arrows in Figure 3. This situation scarcely changed when MB was added in the polymerization systems.

\section{Precipitation Polymerization of AAm with MAc and $M B A A m$}

Addition of MAc in the polymerization system altered the mode of particle formation and growth drastically. The SEM views of products in the precipitation polymerization of AAm, MB, and MAc are shown in Figure 4. Fine particles were obtained instead of coarse aggregates when MAc was used above a certain concentration. The increase in the concentration of MAc decreased the size of particles and increased total area of particle surface as shown in Figure 5. This result implied that MAc served as a stabilizer for basic particles. To confirm this, precipitation polymerization of AAm was carried out in the presence of poly-MAc. Very fine microspheres were obtained from this polymerization as expected. In these systems, the particles are supposed to be stabilized mainly by steric stabilization effect because of the amphiphilic property of MAc units. Electrorepulsive force may somewhat contribute to the stabilization even in such organic medium as proposed in polymerization of vinyl chloride. ${ }^{8,10}$

The manner for particle growth was observed with an electron microscope. The result is shown in Figure 6. Contrast to the case of precipitation polymerization of AAm alone, particles were always monodisperse and grew up gradually with increasing conversion

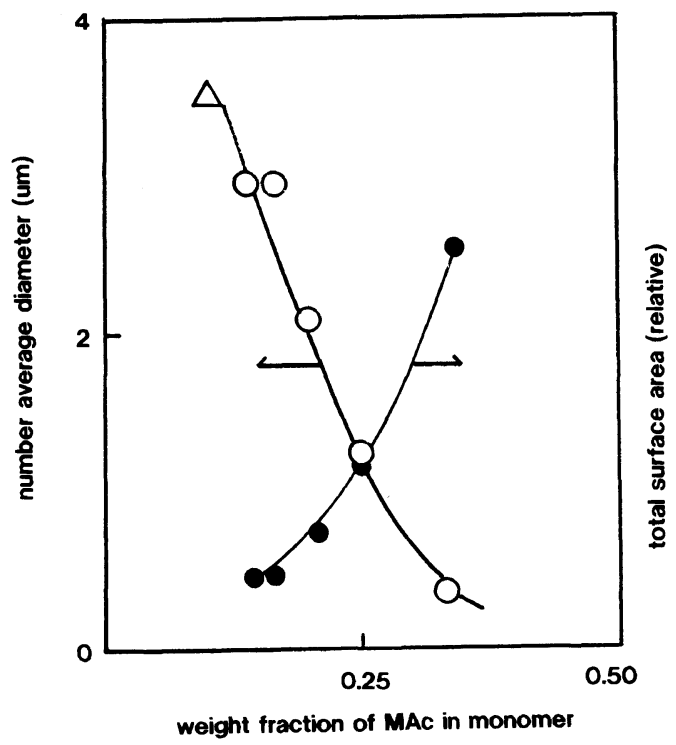

Figure 5. Dependence of particle size ${ }^{1}$ and total surface area of particles on MAc content in monomer feed. 1: the particle size is a hydrodynamic one in water measured by photon correlation spectroscopy; Polymerization: AAm/ $\mathrm{MB} / \mathrm{MAc} /$ ethanol/AIBN $=x(1 \sim 8) / 1 / 1 /(48-x) / 0.025(\mathrm{~g})$. 


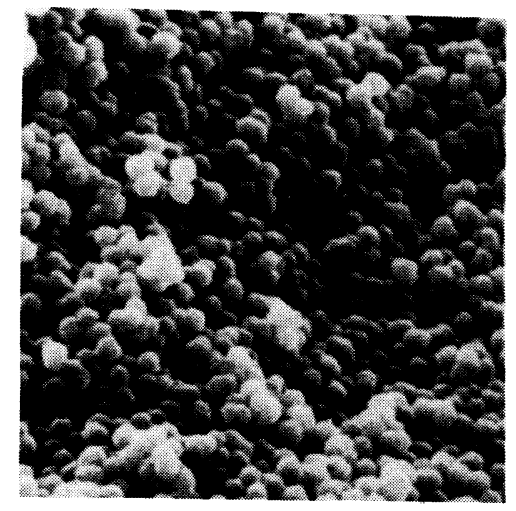

conv. $5 \%$

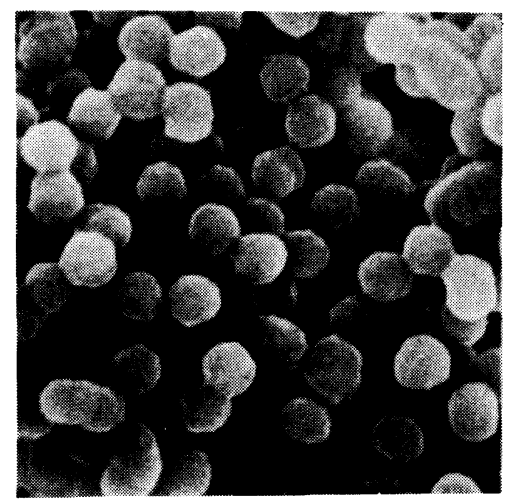

conv. $48 \%$

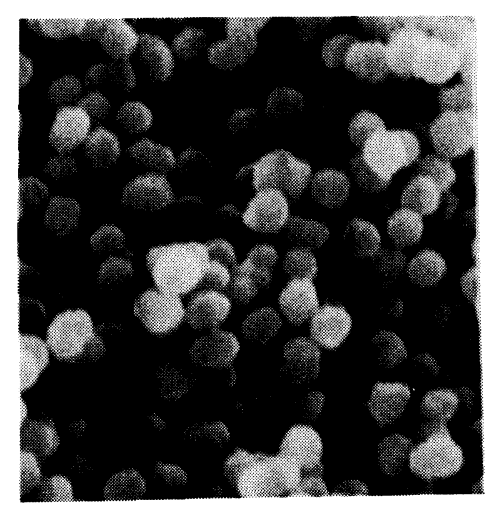

conv. $25 \%$

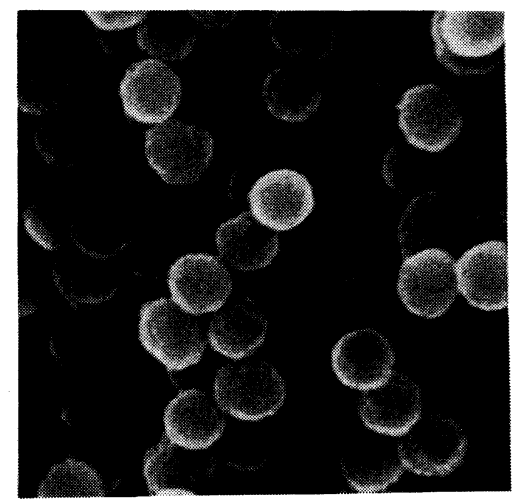

conv. $100 \%$

\section{$1 \mu \mathrm{m}$}

Figure 6. SEM views of polymer particles at different conversions in the precipitation copolymerization of AAm, MAc, and MB in ethanol. Polymerization: AAm/MB/MAc/ethanol/AIBN=4/1/1/44/0.025 (g).

in the copolymerization. No newly-formed basic particles were found in the course of polymerization after $5 \%$ conversion. These facts indicate that the number of particles was fixed at the very early stage of polymerization in AAm-MAc copolymerization system. This could be confirmed quantitatively by the proportionality of the diameter with (conversion $)^{1 / 3}$ as shown in Figure 7. This situation is very similar to that of soap-free emulsion polymerization in which narrow particle size distribution results.

It is believed in soap-free emulsion polymerization that the characters, such as stability and extent of swelling, of basic particles formed in the early stage of polymerization decide the monodispersity of final products ${ }^{11}$. The stability of AAm-MAc particles has been discussed. Now, swellability of AAm-MAc copolymer with ethanol and AAm monomer 


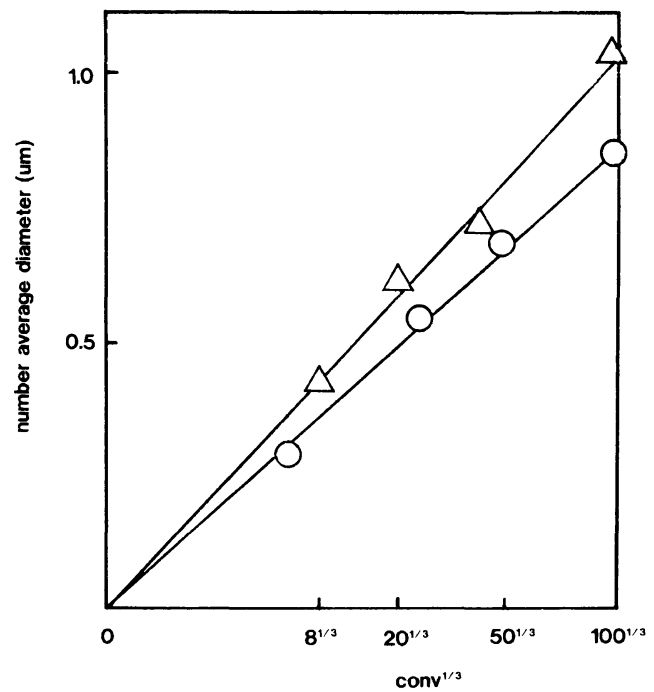

Figure 7. Relation between diameter ${ }^{1}$ of particles and (conversion) $^{1 / 3}$ in two precipitation copolymerization systems. AAm/MB/MAc, $\bigcirc: 4 / 1 / 1, \triangle: 4 / 1 / 0.5$ (g). 1: the diameter was measured by SEM of dried particles.

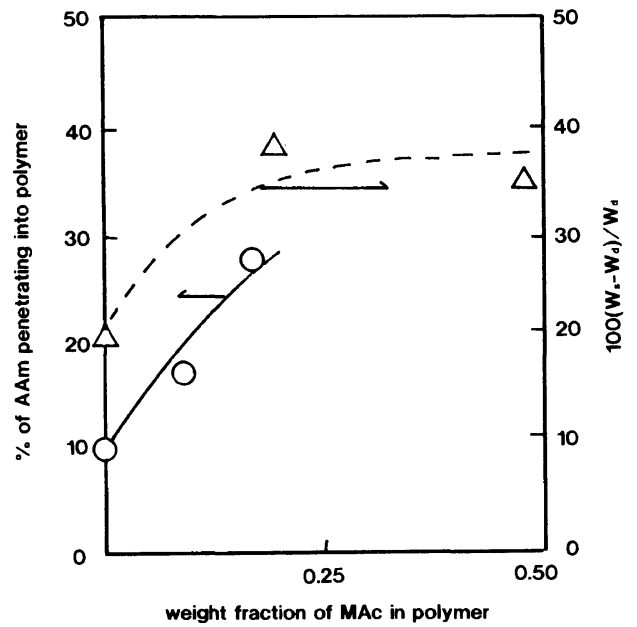

Figure 8. Swelling of polymers with ethanol and monomer as a function of composition of polymer. $O$ : fraction of AAm penetrating into polymer; $\triangle:\left(W_{\mathrm{s}}-W_{\mathrm{d}}\right) /$ $W_{\mathrm{d}}$ in which $W_{\mathrm{s}}$ and $W_{\mathrm{d}}$ are the weights of polymer swollen with ethanol and dried polymer, respectively.

is discussed. The measurement of swellability was carried out using some copolymers having different MAc/AAm fractions. The results shown in Figure 8 indicate that MAccontaining copolymers absorb more ethanol

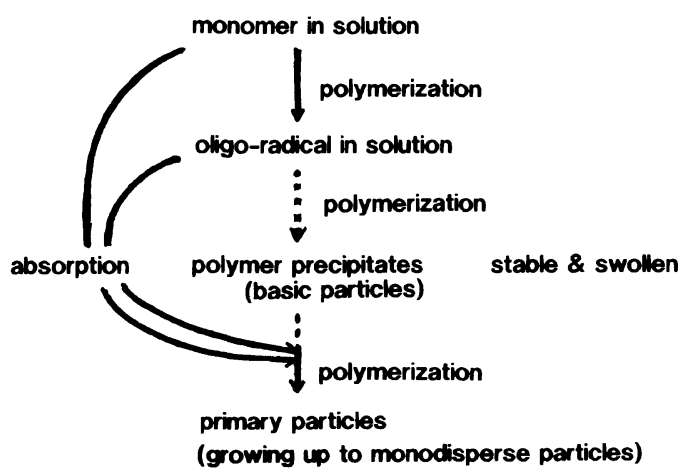

Figure 9. Scheme for particle growth in the precipitation copolymerization of AAm, MB, and MAc in ethanol.

and AAm monomer than a homopolymer of AAm does.

It will be reasonable to consider from the above results that AAm-MAc copolymer particles are different from poly-AAm ones in their high absorbability of oligo-radicals as well as monomer molecules. Accordingly, it is likely that MAc-containing particles formed in early stage of polymerization will scavenge belatedlyformed oligo-radicals and reduce the possibility for the formation of new basic particles. Even if some oligo-radicals escape from the scavenge, the resulting basic particles will have opportunities to coalesce with highly-swollen and soft AAm-MAc particles existing previously. Summarizing these speculations, the scheme for AAm-MAc copolymer particle formation is presented as Figure 9, differing from Figure 3 which was suitable for AAm particle formation. In the precipitation copolymerization of AAm and MAc, the pathway shown by solid arrows in Figure 9 will be dominant for the growth of copolymer particles except for the very early stage of polymerization.

\section{CONCLUSIONS}

Precipitation polymerization of acrylamide in alcohols resulted in the formation of aggregates of primary particles. On the contrary, addition of a certain amount of methacrylic acid in the polymerization system 
gave fine and monodisperse microspheres having a diameter around $1 \mu \mathrm{m}$. This was attributed to the contribution of methacrylic acid units to the stabilization of primary particles and enhancement of absorbability of monomers and dispersant in the primary particles.

\section{REFERENCES}

1. J. W. Vanderhoff, F. V. Distefano, M. S. El-Aasser, R. O'Leary, O. M. Shaffer, and D. L. Visioli, Dispers. Sci. Technol., 5, 323 (1984).

2. W. Baade and K. H. Reichert, Eur. Polym. J., 20, 505 (1984).

3. F. Candau and Y. S. Leong, J. Polym. Sci., Polym.
Chem. Ed., 23, 193 (1985).

4. V. Glukhikh, C. Graillat, and C. Pichot, J. Polym. Sci., Polym. Chem Ed., 25, 1127 (1987).

5. R. H. Pelton and P. Chibante, Colloids Surfaces, 20, 247 (1986).

6. H. Kawaguchi, M. Kawahara, N. Yaguchi, F. Hoshino, and Y. Ohtsuka, Polym. J., 20, 903 (1988).

7. M. W. Allsopp, Pure Appl. Chem., 53, 449 (1981).

8. A. Guyot, Makromol. Chem., Macromol. Symp., 10/11, 461 (1987).

9. F. C. DeSchryver, G. Smets, and J. Van Thielen, Polym. Lett., 6, 547 (1968).

10. E. Diamadopoulos, I. Zoubourtikoudis, and C. Kiparissides, Colloid Polym. Sci., 268, 306 (1990).

11. R. M. Fitch, in "Science and Technology of Polymer Colloids." vol. 2, G. W. Poehlein, R. H. Ottewill, and J. W. Goodwin, Ed., Martinus Nijhoff Publishers, The Hague, 1983, pp. 100-111. 